\title{
MobGeoSen: facilitating personal geosensor data collection and visualization using mobile phones
}

\author{
Eiman Kanjo · Steve Benford · Mark Paxton · Alan Chamberlain · \\ Danae Stanton Fraser · Dawn Woodgate · David Crellin · Adrain Woolard
}

Received: 8 November 2006/ Accepted: 26 June 2007/Published online: 8 August 2007

(C) Springer-Verlag London Limited 2007

\begin{abstract}
Mobile sensing and mapping applications are becoming more prevalent because sensing hardware is becoming more portable and more affordable. However, most of the hardware uses small numbers of fixed sensors that report and share multiple sets of environmental data which raises privacy concerns. Instead, these systems can be decentralized and managed by individuals in their public and private spaces. This paper describes a robust system called MobGeoSens which enables individuals to monitor their local environment (e.g. pollution and temperature) and their private spaces (e.g. activities and health) by using mobile phones in their day to day life.
\end{abstract}

\author{
E. Kanjo $(\bowtie) \cdot S$. Benford $\cdot$ M. Paxton $\cdot$ A. Chamberlain \\ Mixed Reality Laboratory, School of Computer Science and IT, \\ University of Nottingham, Nottingham, UK \\ e-mail: eiman.kanjo@cl.cam.ac.uk \\ S. Benford \\ e-mail:sdb@cs.nott.ac.uk \\ M. Paxton \\ e-mail: mcp@cs.nott.ac.uk \\ A. Chamberlain \\ e-mail:azc@cs.nott.ac.uk \\ D. S. Fraser · D. Woodgate \\ Department of Psychology, University of Bath, Bath, UK \\ e-mail: D.StantonFraser@bath.ac.uk \\ D. Woodgate \\ e-mail: D.Woodgate@bath.ac.uk \\ D. Crellin \\ Abington Partners, 146 London Road West, Bath, UK \\ e-mail: david@auc.co.uk \\ A. Woolard \\ BBC Creative R\&D, New Media and Technology, London, UK \\ e-mail: Adrian.woolard@bbc.co.uk
}

The MobGeoSen is a combination of software components that facilitates the phone's internal sensing devices (e.g. microphone and camera) and external wireless sensors (e.g. data loggers and GPS receivers) for data collection. It also adds a new dimension of spatial localization to the data collection process and provides the user with both textual and spatial cartographic displays. While collecting the data, individuals can interactively add annotations and photos which are automatically added and integrated in the visualization file/log. This makes it easy to visualize the data, photos and annotations on a spatial and temporal visualization tool. In addition, the paper will present ways in which mobile phones can be used as noise sensors using an on-device microphone. Finally, we present our experiences with school children using the above mentioned system to measure their exposure to environmental pollution.

Keywords Environmental monitoring . Mobile computing · GPS positioning

\section{Introduction}

Mobile phones have become ubiquitous within our everyday life. Modern mobile phones are capable of capturing high resolution images and of recording high quality sounds. Many are also equipped with a high-resolution color display; they support different wireless networking standards and have sufficient memory to run a variety of different applications. The development of software applications for mobile computers has been recently spurred on by the availability of more powerful operating systems and the transfer of standardized programming languages on ever-smaller computing platforms. People take their phones 
everywhere, using them in a variety of environments and situations to perform a whole range of different tasks. Mobile phones provide an opportunity to monitor local environments in order to monitor and reduce pollution, have medical applications and be used to tackle other problems on a societal scale. This is because of their market that relates to the sheer numbers that are in daily use [5]. The key to using mobile phones for such applications is based around the use of their built-in sensing capabilities [7, 13]. These include; cameras, GPS sensors and microphones. When these devices are used with affordable off-the-shelf environmental sensors and location tracking devices there are a wide range of application to which these systems can be applied.

Although using mobile phones for environmental monitoring purposes will provide new services, they will also create new challenges and thus create new research trends in pervasive computing. In this paper we introduce an alternative, affordable and efficient prototype system for the collection of environmental information by ordinary people. These users can take their phones where ever they go and thereby use them in various environments and situations to perform different tasks. At this stage we are not concerned with issues of large scale network communication or data management. Primarily the focus is on the collection of data and its manipulation by individuals who own mobile phones and are interested in the environmental changes occurring in their surroundings.

Our prototype system, known as "MobGeoSen", it consists of a series of software components and off-theshelf wireless devices: a GPS receiver and sensor datalogger used in conjunction with a mobile phone. The software components provide a robust, expandable platform for mobile data collection (from environmental sensors), including on-device gathering, refining and the integration and interpreting of data for a variety of applications. These components are:

1. A sound level sensor that uses the phone's microphone. This is an important feature of the system as it allows for the evaluation of one of the phone's key built-in sensor capabilities.

2. A framework for multiple Bluetooth connections. This allows the datalogger and the GPS to wirelessly and simultaneously connect to the phone.

3. When connected to the GPS, the ability to geotag (images, annotations) the collected data and transmit them (to a GIS for example).

4. Coupling the location data, the sensor data, photos and annotation into a KML file format for further use with Google Earth 3D visualizations of the data on the PC.

Clearly, a vital element of such a system will be the protection of users' privacy, although we suggest that maintaining the users' ability to retrieve or identify their own data may nevertheless be an important design requirement.

This paper discusses the research and development of the various software components that the MobGeoSen system comprises of. The sensor technologies and applications that were developed are then described and discussed. The paper then presents the tests that were carried out by the users of the system at the schools; however, another paper will focus upon the resulting interaction design challenges related to the system.

Finally, sensor system design issues concerned with the use of sensor data are discussed. Details of the specific environmental applications for which the system prototype were originally designed and its use with Google Earth.

\section{Environmental monitoring}

Currently, many of the systems used for collecting environmental parameters are based on sensor network nodes which are located in a specific local regions that transfer data to a central database (iMotes [6], ECOs [11] and BTnodes [1]). The large scale of deployment and their price requirements imply that resources in terms of energy, memory, computational speed and bandwidth are severely constrained. In many cases, sensor data is collected from static points. Therefore, most wireless sensor network (WSN) kits have been designed either to demonstrate novel wireless sensor network architectures (e.g. [4, 8]) or for industrial applications [2].

A variety of projects have shown how the integration of sensors and positioning technologies used in conjunction with mobile computing devices can support data collection for environmental applications, without the overheads and complexities of wireless sensor networks. Projects such as $[12,14]$ have considered the embedding of direct awareness in mobile devices which is boosted by the rapid advance in sensor technology.

Gellersen et al. [3] used diverse sensors aimed at the direct acquisition of multi-faceted information from the local environment [3], while others [9] used ubiquitous devices such as PDAs and portable computers linked to an electronic sensor board using the serial port. Steed et al. [14] built a data collection system which can be carried by users to show local variations in pollution levels. Vivoni [16] developed a data collection toolkit to assist environmental scientists and improve the collection of field data associated with the location data [16].

There has been little research into data collection using mobile phones with sensors embedded, even though this approach offers several potential advantages.

Firstly, mobile phones are carried by a large percentage of the population (in many countries), secondly, data 
collection using mobile phones should be more energy efficient, because a mobile collector can directly pick up the data from the sensor instead of having to send the data across an entire sensor network. Thirdly, mobile phones can be used to sense, process, store and transfer contextual data such as photos and messages. Modern mobile phones also have various communication channels which are capable of transferring the data remotely to other devices.

Individuals could install sensors in their private space (to observe their home or their possessions) or on themselves (to monitor their health, their physiological condition). There may be people who are interested in monitoring their environmental conditions. The sensors could be placed indoor and outdoors. Levels relating to temperature, sound, motion and pollutants in the environment could be observed. A group of neighbors may collectively decide on a kind of data collection action, to perhaps measure sound levels from a nearby road or track congestion along side streets in the area. It is also possible that geographically dispersed communities will form around data collection, as do plane spotters, for example. It may also be helpful to scientists who donot have access to a sensor network and want to carry on some measurements and map some environmental parameters.

\section{The MobGeoSens system}

\subsection{System overview}

The MobGeoSen data collection system consists of three Bluetooth-enabled devices capable of acquiring, storing, displaying, transmitting environmental contextual and geopositional data. These are Nokia series 60 camera phones, a GPS receiver and sensor datalogger.

GPS sensors have become available in very small package sizes and with Bluetooth capabilities enabling their integration in mobile devices. The GPS that we have used for the prototype is a Bluetooth-enabled Haicom 406B. This uses the new SIRF STAR III technology that supports connections with up to 20 satellites at one time, rather than the 12 channels of older devices. It also has $8 \mathrm{~h}$ of battery life and has a built-in active antennae. The GPS receiver can transmit a position as WGS84 latitude, longitude at high speed $(115,200 \mathrm{kbps})$, however, we set the system to output one location report each second. The GPS receiver also supplies accurate time for the logs.

The datalogger is a ScienceScope ${ }^{1}$ Logbook WL, which is a sophisticated Bluetooth enabled logbook with LCD display and 8-file memory for remote use. It can be connected with up to four sensors at the same time, for

$\overline{{ }^{1} \mathrm{http}: / / \mathrm{www}}$.ScienceScope.co.uk. example it can measure simelteneously levels of carbon monoxide, temperature, acceleration and electric field strength. It also gives the user the option of wireless or USB connection and $8 \mathrm{~h}$ of continuous wireless datalogging.

The system has two structural modes that are responsible for querying sensors and GPS periodically (currently once per second) and returning that data for processing and storing. In the first mode (data-logger mode), the system acquires sensor data from the datalogger and location data from the GPS at the same time via a Bluetooth connection, as shown in Fig. 1. In the second mode (sound sensor mode) the system acquires sound levels in the surrounding environment using the phone's built-in microphone. This component only connects the GPS to the phone since the sound level is measured internally by the phone (as shown in Fig. 1).

The phone is also equipped with a camera. This allows the users of the system to take geo-referenced photos that can be displayed automatically by using Google Earth. As the current prototype has no built in wireless networking, data upload is only possible when the device is synchronized with a desktop using Nokia PC Suite via Bluetooth, infrared or USB connection.

Figure 2 illustrates the architecture of the system, identifying how the separate hardware elements within the phone and the external sensor components fit together.

MobGeoSens may collect data from three sources, external devices connected via Bluetooth, the microphone built in to the phone and annotation data entered by the user. This can consist of text and photographs. Each of these methods are further expanded upon in the following section.

\subsection{Data collection from external Bluetooth enabled sensors}

In order for the phone to simultaneously collect sensor data from the datalogger and location from GPS, an advanced component was developed which establishes multiple Bluetooth device connection and communicates over those connections.

The Multiple Bluetooth device connection is a generic component which provides the following capabilities. This scans for Bluetooth devices within range. Service discovery then asks the discovered Bluetooth devices for the services they offer, filtering the service discovery query to return only the service class required. Most Bluetooth devices support the Bluetooth service called "serial port profile" which emulates a serial cable, eliminating the need for a cable between the device and the host device.

Bluetooth GPS and datalogger act as slaves and wait for other devices such as the mobile phone to connect to it; it 
Fig. 1 System components in two modes: (left) data logger mode and (right) sound sensor mode
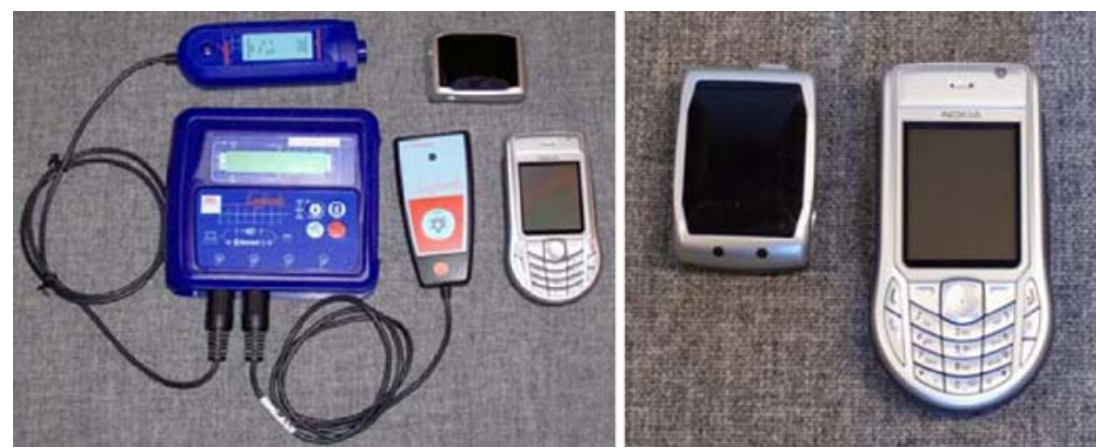

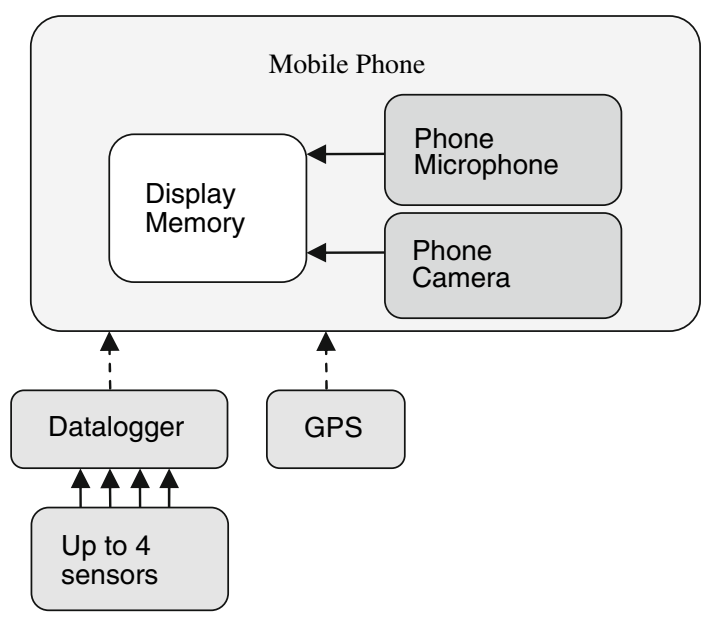

Fig. 2 Overview of system architecture

does not actively go out and make the connection itself. Because of this, the service discovery query will only return the service entries that match the service class of the GPS and the LogBook. A connection will then be established to both of the Bluetooth devices. Once the connections are established, messages can be sent from the master device to the connected slaves to start reading data. Figure 3 shows a screenshot of the phone interface with a number of sensor reading.

\subsection{Sound level collection using a phone's microphone}

Environmental noise is unwanted sound from a variety of different sources. Noise may emanate from: traffic, construction work, aircraft, sports events, leisure parks or domestic noise. Each noise has different characteristics and poses specific problems, for example noise can annoy, disturb and affect the health of people of different age groups [10].

The sound sensor application turns the mobile phone into a low-cost data logger for monitoring environmental noise. It uses the phone's microphone to assess sound levels in the surrounding environment. The software

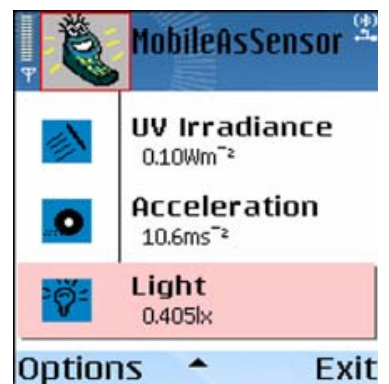

Fig. 3 Screenshot of the phone interface

combines the sound data with the external GPS receiver data in order location data in order to generate a map of sound levels encountered during a journey.

The sound monitor component also displays sound level data as a real-time graph of sound measurment and satellite information as shown in Fig. 4. In this screenshot it was very quiet until a radio is turned on so the user could listen to the news. When the news reader speaks the sound level varies, dropping to a low level during breaks in the speach.

\subsection{User interface for adding geocoded annotations and placemarks}

The software prompted the users to add geocoded annotations and placemarks during their journey; for example, users were asked for their names and their destination before they start a data collection journey. They could also add place-marks and annotations at will, as shown in Fig. 5.

In integrating the phone's built in camera into the application at the point of collection, images could be embedded in the data log with no further action required.

This meant that the images could be automatically placed in-location when the user visualized the data.

The Nokia phones used in this research had a built-in phone camera with a resolution of $640 \times 480$. In order to minimize the storage space for these images, the photos taken were saved with a resolution of $320 \times 240$ pixels on the prototype. 


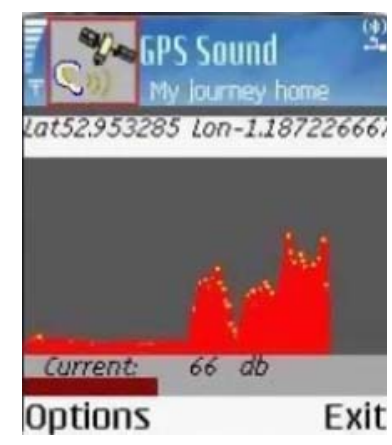

Fig. 4 The first iteration of the on-device visualization. This allowed the user to see a data/time graph whilst collecting data

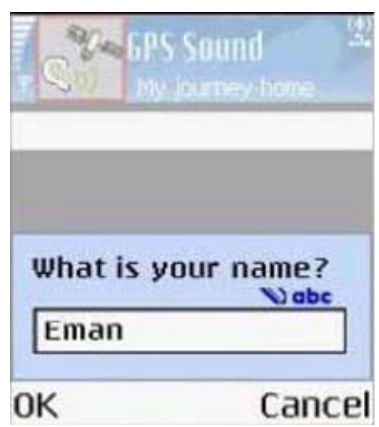

Fig. 5 Screenshot showing the input of an annotation for a geotag

\section{Visualization using Google Earth}

Data is the basic form of information that needs to be collected, managed, mined and interpreted to create knowledge. Discovering the patterns, trends, and anomalies in massive data sets is a challenge.

The first step taken in analyzing the collected data was through the use of visual analysis tools. The aim was to provide end users with a tool that allowed them to monitor how sensor data builds up over time and space. This also provided them with tools that enabled them to interpret the readings from different sensors and positioning technologies.

To support such analysis, a tool was developed that recorded data from a GPS receiver, and on phone sound sensor and the datalogger. This was placed into a text file in the phones' memory. This data (sensor reading, location, waypoints, annotations, photos and time) are saved to a time stamped KML file (Keyhole Markup Language), which is an XML-based language for managing threedimensional geospatial data in the program Google Earth ${ }^{2}$.

Google Earth is used to visualize 3D trails of the data after they had been generated by the phone application, enabling users to review the data they gathered throughout

\footnotetext{
2 http://www.earth.google.com/kml/.
}

a journey. This was done by saving all the latitude, longitude and sensor data to a time-stamped KML files. The photos and user's annotations are linked to time-stamped KML files which were created when the logging stopped.

To support Google earth visualisation applications the KML files needed be uploaded to a PC. For example, users could use Nokia Suite $^{3}$ to upload the data over USB, Bluetooth or Infrared connections. Log files could only be transferred, if the logging of the path had stopped. Once successfully completed, local logs on the mobile could be deleted by double clicking the KML files. Google Earth opened and displayed the track with the associated photos and annotations.

The data format allowed the users to collect the data in two different spatial-temporal modes:

(1) Temporal-data-collection mode: where the time and the sensor data are stored into a text log file. For example sensors can be placed in a home inconspicuously.

(2) Journey based data collection mode.

For example, the noise level data shown in Fig. 3 was collected by one user on a journey away from the Computer Science building at the University of Nottingham. A peak of $95 \mathrm{~dB}$ can be seen where the user approached a set of traffic lights at a junction of two busy roads (Fig. 6).

\section{Evaluating the system}

\subsection{Pre trial testing}

The system's development overlapped two separate research projects-Mobile phone as sensor and participate, ${ }^{4}$ separate evaluation exercises were carried out, though both involved trials in an iterative manner. The iterative design process continoued across number of sessions all of which took place in the context of school classrooms. The detail of this participatory design process will be described in a further paper. However, the evaluation strategies and findings to date are summarized below.

\subsection{Evaluation in a school environment}

In order to demonstrate the domain and use of MobGeosSen kit the project worked closely with science teachers and children at two schools. A research programme of activities was developed that spanned one school year. The

\footnotetext{
3 http://www.europe.nokia.com/A4144903.

${ }^{4}$ http://www.participateonline.co.uk.
} 


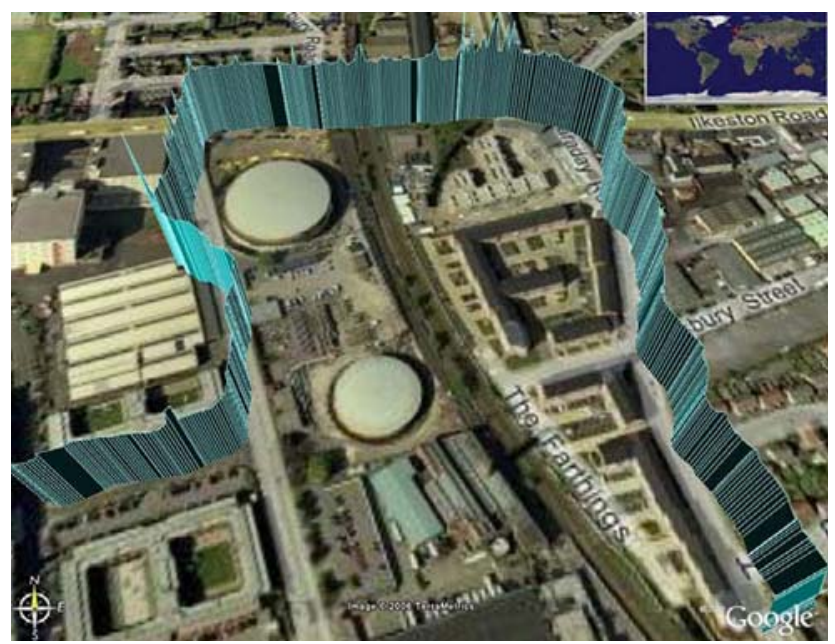

Fig. 6 Google Earth Screenshot showing the sound level during a journey from home to work. The Aerial map overlay used in this image is ${ }^{\odot}$ Getmapping plc copyright

schools are Hayesfield School Technology College in Bath and Castle School in Thornbury near Bristol. The activities were designed to familiarize children with gathering, manipulating, sharing and visualizing two types of pollution data in their environments: $\mathrm{CO}$ and noise. Sixty pupils involved in the participate project were given mobile phones to monitor pollution levels on their journey to and from school over two weeks. In particular, they monitored their exposure to noise and carbon monoxide from transport (see Fig. 7).

The pupils, aged 13-15 years, collected spatially and temporally intensive data streams of carbon monoxide and noise pollution over two weeks, in an attempt to work out what influences pollution levels in their local environment.

The aim of this trial was to encourage school children to learn more about their environment whilst they were doing their everyday activities. Back in the classroom, they were given laptops with Google Earth installed and high resolution maps of their schools areas so that they could view their data alongside photos and placemarks of the locations in which they had collected their readings. They were also able to compare the data they had collected with scientists and members of the public.
Figure 8 shows visualisation of carbon monoxide levels on student's route to sport event including a photo of of nearby busy road collected during the walk (see Fig. 8).

To enable the users to fully understand and use the system a user manual was produced for both groups. This consisted of an overview of the system along with specific step-by-step instructions for the users.

The evaluation of use is carried out by eliciting feedback from the teacher and students. The students engaged well with the trials, and the teacher expressed surprise at the degree of knowledge of technology that some of them possessed, in particular a group of boys who as a rule frequently exhibited problem behaviour, and did not achieve well in class. This was not surprising as a recent study found that $91 \%$ of children by the age of twelve own and use a mobile phone [15]. The class enjoyed trying out both the new sensors linked to mobile phones during their journeies to and from home.

The participants treated the system in a way that suited the tasks and technologies at hand. Especially in the face of technical problems such as GPS errors and power consumption, they focused on the readings that they knew best and did not attempt to make a common and precise sensor map. They also used the camera provided in the system to help record and recount their experience.

Results of Google Earth analysis of the sessions and teacher interviews suggest that this context inclusive approach is significant for three key reasons. Firstly, it allows individuals to reflect on method as part of data collection. Secondly, it provides an aide-memoir for groups of students who have collected data together, when interpreting results. Thirdly, it allows new participants who have engaged in similar processes to understand new perspectives on their own and others' data.

Furthermore, the evaluation also involved a "60 second scientist" filming workshop, managed by our BBC colleagues. Groups of students made short films based around environmental issues generally, and in particular the work that they had carried out. Each group was given a question or theme to start them off, and they then storyboarded their ideas, wrote scripts, shot and edited the footage. Analysis of video data of the groups engaged in making their films
Fig. 7 MobGeoSen used by pupils to collect environmental data
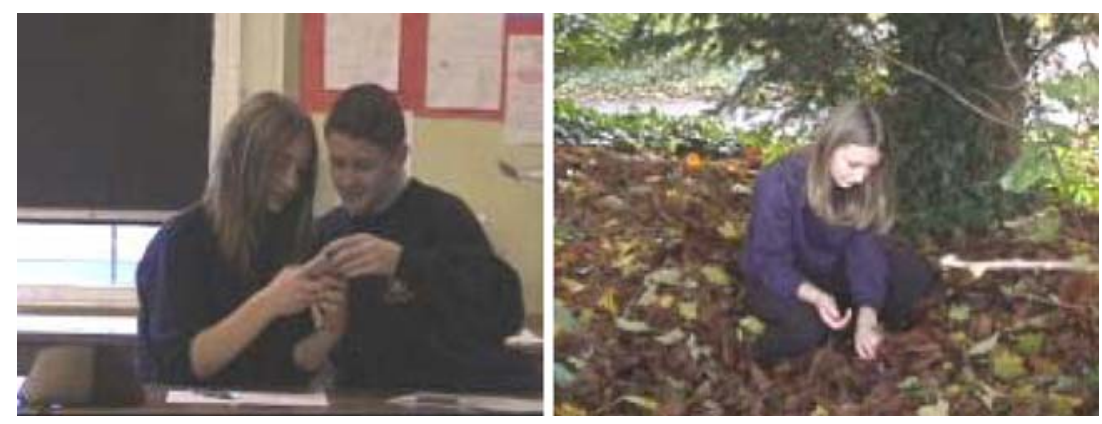


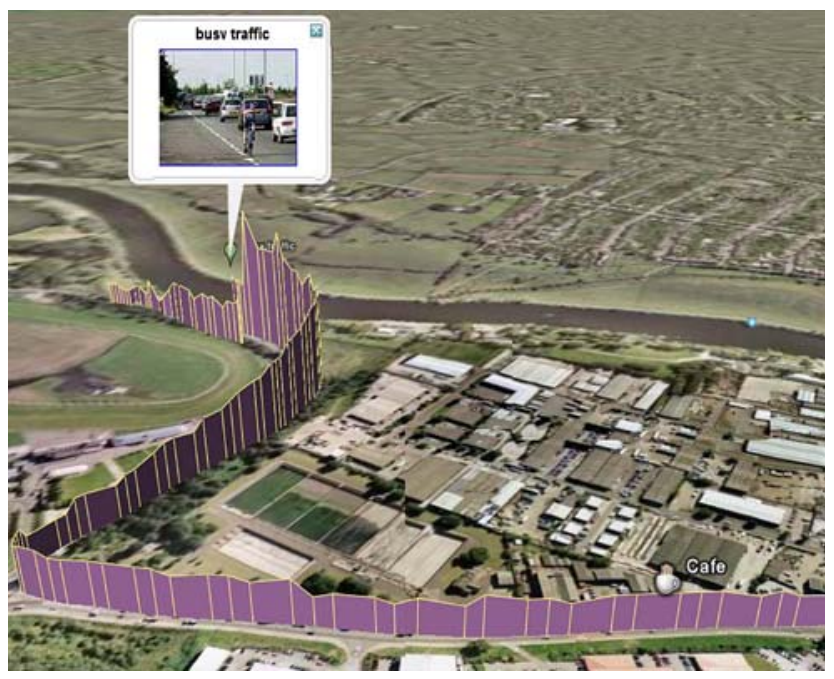

Fig. 8 A trail of carbon monoxide data collected by a student, rendered in Google Earth. The aerial map overlay used in this image is ${ }^{\odot}$ Getmapping plc copyright

indicated that this activity was an effective reflection tool, focusing their minds on the work that they had been doing, promoting discussion, and encouraging them to look for additional information on the topics they had been working on.

We have identified the following scientific activities which are made possible by the use of our system:

- Collecting data. It enables the collection of data that would otherwise be out of the reach of school science learners. Phenomena which would formerly have remained undetected by our unaided senses can now be visualized, and thus made more concrete and understandable to children. For example, carbon monoxide (CO) is an invisible, odourless, toxic gas which does not occur naturally in the atmosphere. One of its sources is the exhaust produced by motor vehicles. New sensor technologies enable us to detect and measure CO quite easily.

- Evaluating and analysing data. It can provide (or prompt recall of) information about the context in which the data were collected, to aid understanding and analysis, and promote discussion. For example, Google Earth, photos, annotations and GPS locations data were collected by students alongside CO measurements, so that the students were able to look at the graphs produced from their data and photos in direct relation to GPS location.

- Authentication of data. It can provide authentication of data, which is important for two reasons. Firstly, accurate and correctly used sensor technologies (which are essentially a simplified version of the same kinds of instruments as those used by professional scientists) create the potential for schools to collect data which are reliable enough to be of use to professional scientists' own investigations (e.g. if their aim is to produce a national picture of some phenomenon). Secondly, data collection using these means can enable school students' work to be situated in a given time and place. The participate data, for instance, is date and time stamped, and pertains to a specific GPS position. This may become an important source of authentication should practical investigations become part of the external examination system. However, issues of privacy and personal safety are also raised.

\section{Technical challenges}

The development of this technology and subsequent testing has highlighted some technical challenges. Three of the main challenges are discussed in the following sections:

\subsection{Battery consumption}

An important factor when conducting experiments on mobile devices is battery consumption. This can be an import issue to consider if the mobile phone additionally has to communicate with devices other than by using a Bluetooth connection, as was the case with MobGeoSen, where the additional devices were the GPS unit and the Data logger.

On standby, the Nokia 6680 phone runs from 6-11 days. However, this phone has a lot of other technologies attached which are a further drain on its power. During continuous usage the battery life is reduced. In our system the mobile phone is the master device which can connects and receives data packets, while in point-to-point mode (as in the case of the GPS only) or in point-to-multipoint mode (as in the case of connection to GPS and the DataLogger). In order to find out the battery consumption of the mobile phone when connected in different connection modes, measurements have been made at intervals of 15 min each. These were made for $8 \mathrm{~h}$ straight. The graph in Fig. 9 shows the battery consumption over time with five different mode settings.

The battery consumption relating to the use of the phone's internal microphone was inconsequential. Also the battery consumption of the Bluetooth connection to the GPS receiver varied over time (not linear). When the Datalogger and GPS connected the battery consumption was the highest. However, it depended on the size of the data sent by each device and on the regularity of the data sent. 


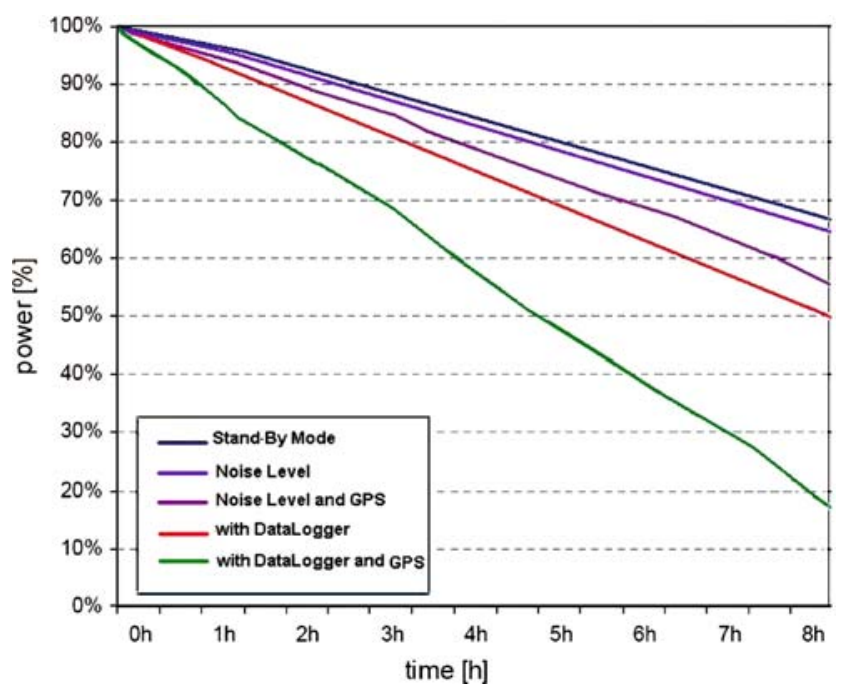

Fig. 9 The battery consumption over time, with five different modes settings

It is important to mention here that taking images with the camera phone can alter the power consumption level sporadically depending how if often it is used.

Although the mobile phone may last for hours and sometimes days, the MobGeoSens cannot work continuously more than $8 \mathrm{~h}$ since the GPS and Datalogger batteries cannot last for more than an $8 \mathrm{~h}$ period.

\subsection{Issues with GPS and Bluetooth connectivity}

The system used a GPS receiver that was powered by SiRF Star III chipset, with 20-channel satellites to give better positional accuracy. However there were some issues relating to GPS and Bluetooth connectivity.

For example, users found it hard to get a GPS fix every time they used the system, since some regions have different number of satellites available. Also Bluetooth connectivity was occasionally lost leading to missing data or errors in the system. However, it is worth noting that connectivity, accuracy and availability issues are being vigorously attacked in the navigation community.

There are plans to improve the system by adding more user messages to the interface to warn them when they lose connection and when they are in a black area with no satellite coverage.

\subsection{Memory/storage}

The amount of data collected over an extended period of time is dependent upon the maximum data storage capacity of the mobile and the battery lifetime.
Table 1 Size of memory used in the experiment

\begin{tabular}{lc}
\hline Start time & 14062006 10:45:51 \\
End time & 14062006 14:46:43 \\
$\begin{array}{l}\text { Number of photos taken } \\
\text { during the journey }\end{array}$ & $\begin{array}{l}15 \text { photos at resolution of } \\
640 \times 480 \text { pixels in JPEG format }\end{array}$ \\
$\begin{array}{l}\text { Size of photos taken } \\
\text { during the journey }\end{array}$ & $\begin{array}{c}320 \mathrm{~kb} \text { (sizes of images } \\
\text { were between } 18 \text { and } 30 \mathrm{~kb} \text { ) }\end{array}$ \\
$\begin{array}{l}\text { Number of annotations } \\
\text { Size of text log file }\end{array}$ & 9 \\
$\begin{array}{l}\text { Size of KML file } \\
\text { Total size of memory used } \\
\text { for this experiment }\end{array}$ & $365 \mathrm{~kb}$ \\
\end{tabular}

The Nokia mobile phones used in the system have $64 \mathrm{Mb}$ of built in memory and $64 \mathrm{~kb}$ in additional multimedia card.

To test the size of memory available for MobGeoSen we gave the kit to a user to collect sound level data while walking in his local environment. Table 1 shows that the journey has consumed $1.25 \mathrm{Mb}$ of the memory. During the journey, the user has taken 15 images, 7 annotations.

\section{Conclusion}

We have demonstrated the feasibility of developing an environmental sensing toolkit using mobile sensing devices. This enabled new types of monitoring that allowed individuals to monitor their local environment (e.g. variation in pollution) without being part of a large networked system (sensor network). Also, the process of sensing the surrounding environment and sharing the data which raises privacy concerns can be decentralized and managed by individuals in their private and public spaces.

The mobile sensing system also provides a means to engage learners with their subject material by allowing them to generate personalised data, then interrogate this data through the use of sophisticated visualisation tools, with their experience embedded as a fundamental part. Furthermore, the approach allows this data to be shared and compared with others, both within a class, school, network of schools or externally with experts and interest groups. Sharing this kind of data creates a rich pool or information, providing not only a resource for further learning but also a common entry point to engage in cooperation and debate with others.

MobGeoSen uses off-the-shelf ubiquitous computing technologies and a newly developed infrastructure prototype to allow the mobile phone to gather data that can then be aggregated and analysed.

The system will be further developed to enable it to be shared or instantiated by other users wanting to monitor their environment. 
The next stage on device construction is to complete the two second-generation prototypes. Infrastructure work will proceed in several directions: improving the performance of the various processes that support data capture, logging and visualisation; integrating a light sensor using the phone camera; and a real-time collaboration system where sensor data is shared in real-time amongst several participants and GPRS for remote data access.

We are currently implementing these in the next iteration of MobGeoSens with the intention of conducting further studies to gauge their effectiveness in the future.

The project focused on creating a toolkit that integrate the tasks of collecting data from environmental and GPS sensors, storing the data and making computations based on the data to make it available for a variety of applications.

Acknowledgments This work is a result of two funded projects: the mobile phone and the sensors project, funded by JISC. The PARTICIPATE research project is a collaboration between the universities of Nottingham and Bath, industrial partners, Science Scope, BBC, BT, Microsoft Research and Blast Theory. It is funded by the Department of Trade and Industry.

\section{References}

1. Beutel J, Kasten O, Mattern F, Romer K, Siegemund F, Thiele L (2004) Prototyping wireless sensor applications with BTnodes. In: Proceedings of the first European workshop on sensor networks (EWSN '04), Zurich, Switzerland, pp 323-338

2. Crossbow Technology Inc., MICA2DOT Wireless Microsensor Mote (2006) Available from http://www.xbow.com/Products/ Product_pdf_files/Wireless_pdf/MICA2DOT_Datasheet.pdf. Cited 10 October 2006

3. Gellersen HW, Schmidt A, Beigl M (2002) Multi-sensor contextawareness in mobile devices and smart artefacts. Mobile Netw Appl 7:341-351
4. Hadim S, Mohamed N (2006) Middleware challenges and approaches for wireless sensor networks. IEEE Distributed Systems, vol 7, no 3, art no 0603-o3001, March 2006

5. Hinckley K, Pierce J, Sinclair M, Horvitz E (2000) Sensing techniques for mobile interaction. In: Proceedings of the 13th annual ACM symposium on user interface software and technology (UIST 2000), ACM Press, New York, pp 91-100

6. Kling RM (2003) Intel mote: an enhanced sensor network node. In: Proceedings of the international workshop on advanced sensors, structural health monitoring and smart structures. Keio University, Japan

7. Leichtenstern K, Alexander De Luca A, Enrico Rukzio E (2005) Analysis of built-in mobile phone sensors for supporting interactions with the real world. PERMID, pp 31-34

8. Moteiv (2006) Moteiv new low cost wireless sensor system, available from http://www.moteiv.com/pr/2006-02-08-tmoteinvent.php. Cited 29 Septemper 2006

9. Munguia ET, Intille SS, Lopez L, Larson K (2006) The design of a portable kit of wireless sensors for naturalistic data collection. In: Proceedings of PERVASIVE 2006. Springer, Berlin

10. Noise Pollution (2006) Available from http://www. environmentagency.gov.uk/yourenv/eff/1190084/pollution/399148/ ?lang=_e. Cited 10 October 2006

11. Park C, Liu J, Chou PH (2005) Eco: an ultra-compact low-power wireless sensor node for real-time motion monitoring. In: Proceedings of the 4 th international conference on information processing in sensor networks (IPSN '05). Sunset Village, UCLA, Los Angeles, pp 398-403

12. Rudman P, North S, Chalmers M (2005) Mobile pollution mapping in the city. In: Proceedings of UK-UbiNet workshop on eScience and ubicomp. Edinburgh

13. Schmidt A, Aidoo K, Takaluoma A, Tuomela U,Van Laerhoven K, Van de Velde W (1999) Advanced interaction in context. In: HUC '99: Proceedings of the 1st international symposium on handheld and ubiquitous computing. Karlsruhe, Germany, pp 89101

14. Steed A, Spinello SB, Croxford BC, Greenhalgh C (2003) e-Science in the streets: urban pollution monitoring. In: UK e-science all hands meeting

15. The mobile life youth report. http://www.mobilelife2006.co.uk/

16. Vivoni ER, Camilli R (2003) Real-time streaming of environmental field data. Comput Geosci 29:457-468 